\title{
Monte Carlo Algorithm for the Evaluation of the Distance Estimation Variance in RSS-Based Visible Light Positioning
}

\author{
Nobby Stevens \\ DraMCo, ESAT \\ Technology Campus Ghent \\ KU Leuven, Belgium \\ nobby.stevens@kuleuven.be
}

\author{
David Plets \\ WAVES, INTEC \\ Ghent University, Belgium \\ david.plets@ugent.be
}

\author{
Lieven De Strycker \\ DraMCo, ESAT \\ Technology Campus Ghent \\ KU Leuven, Belgium \\ lieven.destrycker@kuleuven.be
}

\begin{abstract}
In this work, the Monte Carlo algorithm to determine the variance on the distance estimation in Received Signal Strength-based visible light positioning is considered. The method is build on the maximization of the signal-to-noise-ratio by means of matched filtering, and leads to a number of characteristics that are typically only obtained after intensive analytical elaborations. It is shown that the results match those obtained by calculating the Cramér-Rao lower bound when only the noise is considered as non-deterministic. It is demonstrated that the method is also applicable when multiple physical parameters exhibit a probability distribution, leading to an assessment of the distance estimation accuracy in more realistic settings.
\end{abstract}

\section{INTRODUCTION}

With the ubiquitous presence of light emitting diodes (LEDs), an emerging technology to deploy artificial light as a carrier for information by intensity modulation is at the horizon. This technology is called Visible Light Communication (VLC) [1]. The light is intensity modulated (IM) and directly detected (DD) by a photodiode or camera (called IM/DD), where the frequency content of the signal is beyond the bandwidth of the human eye in order to prevent flickering. VLC is considered as one of the key technologies for the deployment of the fifth generation of wireless systems $(5 \mathrm{G})$ to support the increased data rates in indoor premises [2].

Indoor positioning is a longstanding research challenge. On one hand, there is the need for a solution of accurate localization of robots in warehouses.
On the other hand, due to the increased usage of personal mobile devices, navigation by individuals in large public areas such as airports, train stations and museums will create enhanced user experience. With the presence of dense, LED-based illumination networks, VLC could be used to provide accurate indoor positioning, better known as Visible Light Positioning (VLP) [3]. The foundations of this solution are sound: calculation of the Cramér-Rao lower bound results in subcentimeter accuracy [4].

In this work, we construct a model to evaluate numerically the variance on the distance measurements in VLP where the received signal strength (RSS) is used to estimate the channel attenuation. The model contains a large number of parameters, allowing to tune the input to a specific configuration. In contrast with other authors (e.g, [5]), we did not elaborate analytically the equations. The approach presented here is Monte Carlowise, based on the creation of a set of normally distributed values of the received signal, where the distribution of derived channel parameters such as the distance is easily determined. In reality, other physical parameters such as the optical power emitted by the LED, the vertical distance $h$ between the LED and observation plane, the responsivity of the photodiode, ... can also obey a certain probability distribution. The described method allows the inclusion of these distributions in combination with the noise process. This leads to more realistic results of the modeling, enhanc- 
ing the predictability of the distance estimation accuracy in realistic scenarios.

This work is organized as follows. In section II, the system is described and the different parameters involved in the configuration under study are presented. In section III, the outcome of the numerical simulations is shown. It is demonstrated that this approach leads to the same results as the analytical derivation of the Cramér-Rao lower bound. As a representative example, it is demonstrated how the distance estimation is influenced when on top of the noise, the receiver height $h$ is also non-deterministic. Finally, section IV summarizes the main findings.

\section{SYSTEM DESCRIPTION}

In Fig. 1, the configuration under study is shown. A ceiling-attached LED with a Lambertian radiation pattern of order $m$ is transmitting a signal $s(t)$ during a time slot with duration $T_{s}$, where $s(t)$ is the time-dependent emitted optical power. At the receiving photodiode, only a fraction of $s(t)$ is impinging the photodiode surface. The ratio between the received optical power and the emitted optical power is determined by the channel attenuation $\alpha$ and defined by (1). $\alpha$ is determined by $m$, the surface of the photodiode $A_{R}$, the known height $h$ of the LED with regard to the plane of the mobile node and the distance $d$ between the LED and the receiver [5]. Here, it is supposed that the LED is pointing downwards and that the photodiode points upwards.

$$
\alpha=\frac{(m+1)}{2 \pi} \frac{h^{m+1}}{d^{m+3}} A_{R}
$$

The received optical power $\alpha s(t)$ is translated into a current by the responsivity of the photodiode $R_{p}$, expressed in $\mathrm{A} / \mathrm{W}$. The received signal $r(t)$ in (2) is non-deterministic due to the presence of additive white gaussian noise $n(t)$ (AWGN) with single side spectral density $N_{0}$.

$$
r(t)=\alpha R_{p} s(t)+n(t)
$$

It is well known that matched filtering maximizes the signal to noise ratio at the receiver side [6]. Applying this method, we have at the output of the matched filter the scalar $r$ at consecutive time

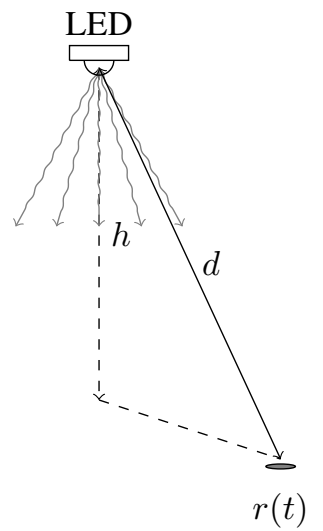

Fig. 1. The LED transmits the signal $s(t)$ during $T_{s}$, while the photodiode receives the noise-polluted signal $r(t)$.

slots $T_{s}$, as formulated by (3). Remark that any timing or synchronization error is excluded.

$$
\begin{aligned}
r & =\int_{T_{s}} r(t) s(t) \mathrm{d} t \\
& =\alpha R_{p} \mathcal{E}+\int_{T_{s}} n(t) s(t) \mathrm{d} t \\
& =\alpha R_{p} \mathcal{E}+n
\end{aligned}
$$

$\mathcal{E}$ is defined as in (4).

$$
\mathcal{E}=\int_{T_{s}} s^{2}(t) \mathrm{d} t
$$

As stated before, $s(t)$ is the optical power transmitted by the LED, so $\mathcal{E}$ is expressed in $\mathrm{W}^{2} \mathrm{~s}$. Considering the properties of $n(t)$ as AWGN, we have that $\mathbb{E}\{n\}=0$ and $\mathbb{E}\left\{n^{2}\right\}=\mathcal{E} N_{0}$. Having determined the mean and variance of $r$, the probability density distribution $p(r)$ is described in (5).

$$
p(r)=\frac{1}{\sqrt{2 \pi \mathcal{E} N_{0}}} e^{-\frac{\left(r-\alpha R_{p} \mathcal{E}\right)^{2}}{2 \mathcal{E} N_{0}}}
$$

Every $T_{s}$, a sample of the distribution $p(r), \hat{r}$ as an estimation of $r$ is obtained. As a result, we have an estimation of the channel attenuation $\hat{\alpha}$ :

$$
\hat{\alpha}=\frac{\hat{r}}{R_{p} \mathcal{E}}
$$

Based on the value of $\hat{\alpha}$ and using (1), the distance $\hat{d}$ (as an estimation of $d$ ) can be calculated as in (7).

$$
\hat{d}=\left[\frac{(m+1)}{2 \pi} \frac{h^{m+1}}{\hat{\alpha}} A_{R}\right]^{\frac{1}{m+3}}
$$


When three or more distance estimations are found, classical methods such as a least-squares estimation or an interative solver for trilateration challenges can be used to find the location of the mobile node [7]. It is clear that if $\hat{r}$ equals the mean value $\alpha R_{p} \mathcal{E}$, that $\mathbb{E}\{\hat{d}\}=d$. The variance on the distance estimation $\sigma_{d}^{2}$ is formulated by (8).

$$
\sigma_{d}^{2}=\mathbb{E}\left\{(d-\hat{d})^{2}\right\}
$$

When performing a purely analytical approach in order to assess the variance on the distance estimations, the formulation of the Fisher information matrix and subsequently the determination of the Cramér-Rao lower bound has to be performed [4], [5], [8]-[10]. It is demonstrated in next section that by executing a Monte Carlo simulation of the model described by (3), the value of $\sigma_{d}^{2}$ equals the Cramér-Rao lower bound $C R L B$ (or $\sigma_{d}=$ $\sqrt{C R L B})$. This method involves the creation of a large set of samples $\hat{r}$ taken from the normal probability distribution $p(r)$ defined by (5). Each $\hat{r}$ leads to a $\hat{d}$ (see (7)), allowing the numerical evaluation of $\sigma_{d}^{2}$ as in (8).

The Monte Carlo approach offers an important advantage with regard to the analytical elaboration in the sense that it is convenient to evaluate the impact of additional distributions on top of the AWGN process. In (3), all parameters are deterministic with the exception of the noise. In reality though, every parameter expressing a physical property has a certain distribution. For example, the observation plane will never be exactly flat, variations of the observation height $h$ in the order of millimeters are reasonable. By performing a Monte Carlo simulation, this phenomenon can be taken into account, in combination with the nondeterministic noise as will be demonstrated in subsection III-B.

\section{RESUlts}

\section{A. Equivalence with the Cramér-Rao lower bound}

In order to focus the attention, we have chosen following representative system parameters: $h=3 \mathrm{~m}, A_{R}=10^{-4} \mathrm{~m}^{2}, R_{p}=0.4 \mathrm{~A} / \mathrm{W}$ and $N_{0}=1.33610^{-22} \mathrm{~A}^{2} / \mathrm{Hz}$ (identical as in [8]). The LED, with a Lambertian radiation pattern of order $m$ equal to 1 , is transmitting $s(t)$ at a frequency of $1 \mathrm{kHz}\left(=1 / T_{s}\right)$. As an example and similar to

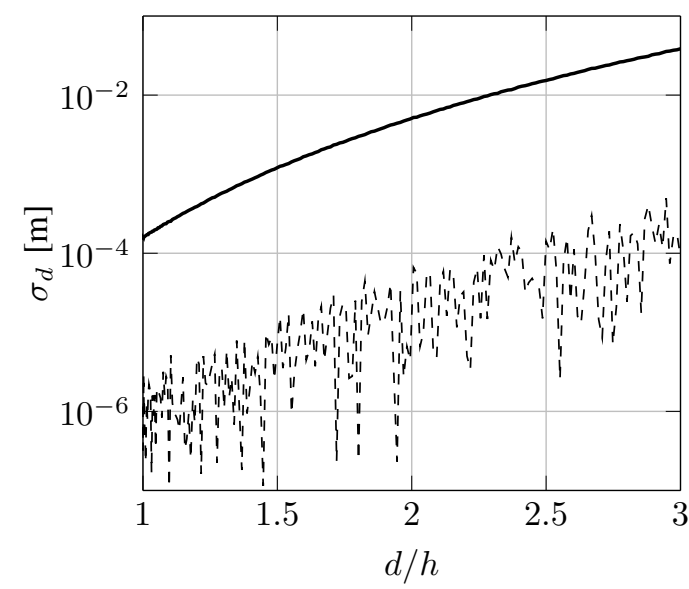

Fig. 2. The black line represents the standard deviation $\sigma_{d}$ as a function of the normalized distance $d / h$, while the dashed line shows the magnitude of the difference with the Cramér-Rao lower bound when $10^{4}$ samples were taken at each normalized distance. The following parameters are applied: $m=1, N_{0}=$ $1.33610^{-22} \mathrm{~A}^{2} / \mathrm{Hz}, h=3 \mathrm{~m}$ and $\mathcal{E}=1.510^{-3} \mathrm{~W}^{2} \mathrm{~s}$.

other work [8], we have chosen $s(t)$ as a raised cosine signal with amplitude of $A$ as in (9).

$$
s(t)=\frac{A}{2}\left[1-\cos \left(\frac{2 \pi t}{T_{s}}\right)\right]
$$

For this raised cosine signal $s(t)$, the average emitted optical power $P_{a v}$ is found by (10), while $\mathcal{E}$ is calculated by (11).

$$
\begin{gathered}
P_{a v}=\frac{1}{T_{s}} \int_{T_{s}} s(t) \mathrm{d} t=\frac{A}{2} \\
\mathcal{E}=\int_{T_{s}} s^{2}(t) \mathrm{d} t=\frac{3 A^{2} T_{s}}{8}=\frac{3}{2} P_{a v}^{2} T_{s}
\end{gathered}
$$

Suppose that the LED is transmitting an average optical power of $1 \mathrm{~W}$, thus leading to a value of $1.510^{-3} \mathrm{~W}^{2} \mathrm{~s}$ for $\mathcal{E}$ since $T_{s}$ equals $1 \mathrm{~ms}$. The resulting dependency of $\sigma_{d}$ on $d / h$ is shown in Fig. 2 , where $10^{4}$ samples of the normal distribution of $p(r)$ (see (5)) were taken. On that same graph, the magnitude of the difference with the Cramér-Rao lower bound is also shown as a dashed line. When the number of samples was further increased, the difference converged asymptotically to zero. This result confirms the correctness of the approach as described. 


\section{B. Addition of the height distribution}

Let us focus on a representative VLP solution to position forklifts within warehouses. Even if the receiver is positioned at the same location for each forklift, it is clear that aspects such as the tire wear, floor irregularities, vibrations, ... make that the value of $h$ corresponds more to a distribution than to an exact value. In order to include this aspect for the distance variance, we have to propose a distribution of the height $h$, as formulated in (12) in case of a gaussian distribution with mean value $h_{0}$ (equal to $3 \mathrm{~m}$ in our example).

$$
p(h)=\frac{1}{\sqrt{2 \pi} \sigma_{h}} e^{-\frac{\left(h-h_{0}\right)^{2}}{2 \sigma_{h}}}
$$

Remark that the described method is not limited to gaussian distributions. Considering the interval $\left[h_{0}-2 \sigma_{h}, h_{0}+2 \sigma_{h}\right]$, about $95 \%$ of all height levels are included. As an illustration, let us suppose that $\sigma_{h}$ equals $2 \mathrm{~mm}$. In Fig. 3, $\sigma_{d}$ is shown for the gaussian distribution of the height in combination with the AWGN and the CramérRao lower bound (where only the AWGN is considered as non-deterministic). The impact of the floor level variance is dominant just below the LED (where $d=h$ ) and converges to the Cramér-Rao lower bound at larger distances. The influence of the height distribution is negligible with regard to the noise for $d / h$ larger than 2 . As a further illustration of the method applicability for non-gaussian probability distributions, we have included as the dashed line the result of another distribution of $h$, where $h$ is uniformly distributed in $\left[3-2.10^{-3}, 3+2.10^{-3}\right] \mathrm{m}$.

\section{CONCLUSION}

In this work, we have constructed a MonteCarlo algorithm-based method to obtain the distance estimation accuracy of RSS-based visible light positioning. It is demonstrated that when only additive white gaussian noise is considered as non-deterministic, the results correspond to the Cramér-Rao lower bound. The method can also be used when other physical parameters obey a probability distribution. As an illustration, we have applied a gaussian and uniform distribution on the distance between the LED and the observation plane $h$. This applicability leads to increased insights concerning the lower limits of the distance

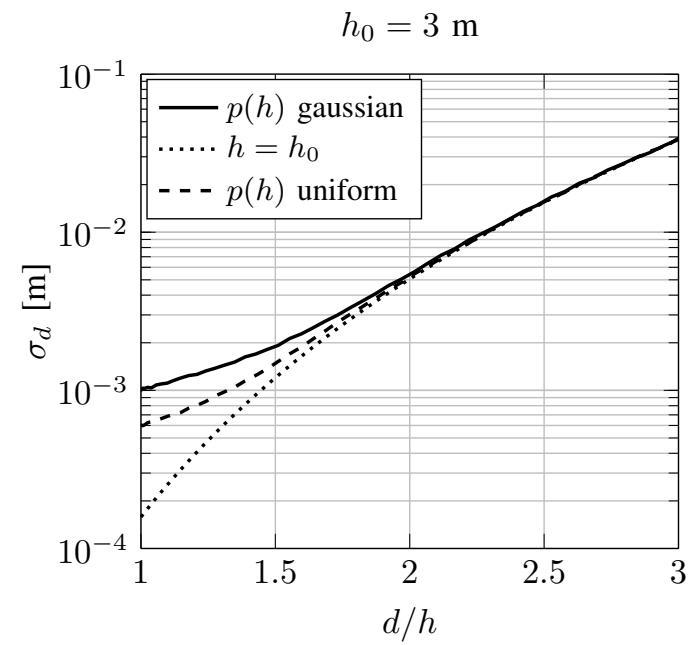

Fig. 3. The black line represents the standard deviation $\sigma_{d}$ as a function of the normalized distance $d / h$ when the gaussian distribution of the height with $\sigma_{h}=2 \mathrm{~mm}$ is taken into account. The dotted line shows the simulation result when only the AWGN is considered, thus presenting the CramérRao lower bound. As a further illustration, the dashed line corresponds to a uniform distribution of the height with a tota width of $4 \mathrm{~mm}$ and center value $h_{0}$. The same parameters as previous result were applied: $m=1, N_{0}=1.33610^{-22}$ $\mathrm{A}^{2} / \mathrm{Hz}, h_{0}=3 \mathrm{~m}$ and $\mathcal{E}=1.510^{-3} \mathrm{~W}^{2} \mathrm{~s}$

estimation accuracy of RSS-based VLP solutions in realistic scenarios.

\section{ACKNOWLEDGMENT}

This work was executed within LEDsTrack, a research project bringing together academic researchers and industry partners. The LEDsTrack project was co-financed by imec (iMinds) and received project support from Flanders Innovation \& Entrepreneurship.

\section{REFERENCES}

[1] A. Jovicic, J. Li and T. Richardson, Visible light communication: opportunities, challenges and the path to market, in IEEE Communications Magazine, vol. 51, no. 12, pp. 26-32, December 2013.

[2] L. Feng, R. Q. Hu, J. Wang, P. Xu and Y. Qian, Applying $V L C$ in $5 G$ Networks: Architectures and Key Technologies, in IEEE Network, vol. 30, no. 6, pp. 77-83, NovemberDecember 2016.

[3] S. De Lausnay, L. De Strycker, J. P. Goemaere, B. Nauwelaers and N. Stevens, A survey on multiple access Visible Light Positioning, 2016 IEEE International Conference on Emerging Technologies and Innovative Business Practices for the Transformation of Societies (EmergiTech), Balaclava, 2016, pp. 38-42. 
[4] X. Zhang, J. Duan, Y. Fu and A. Shi, Theoretical Accuracy Analysis of Indoor Visible Light Communication Positioning System Based on Received Signal Strength Indicator, in Journal of Lightwave Technology, vol. 32, no. 21, pp. 4180-4186, Nov.1, 12014.

[5] T. Q. Wang, Y. A. Sekercioglu, A. Neild and J. Armstrong, Position Accuracy of Time-of-Arrival Based Ranging Using Visible Light With Application in Indoor Localization Systems, in Journal of Lightwave Technology, vol. 31, no. 20, pp. 3302-3308, Oct.15, 2013.

[6] D.A. Guimaraes, Dayan Adione,Digital Transmis sion,Springer Berlin Heidelberg,2009 ,Signals and Communication Technology.

[7] J. Lim, Ubiquitous 3D positioning systems by led-based visible light communications," in IEEE Wireless Communications, vol. 22, no. 2, pp. 80-85, April 2015.

[8] M. F. Keskin and S. Gezici, Comparative Theoretical Analysis of Distance Estimation in Visible Light Positioning Systems, in Journal of Lightwave Technology, vol. 34, no. 3, pp. 854-865, Feb.1, 12016.

[9] H. Steendam, T. Q. Wang and J. Armstrong, Theoretical Lower Bound for Indoor Visible Light Positioning Using Received Signal Strength Measurements and an ApertureBased Receiver, in Journal of Lightwave Technology, vol. 35, no. 2, pp. 309-319, Jan.15, 152017.

[10] E. Gonendik and S. Gezici, Fundamental Limits on RSS Based Range Estimation in Visible Light Positioning Systems, in IEEE Communications Letters, vol. 19, no. 12 , pp. 2138-2141, Dec. 2015. 